\title{
Fluorescent Chemosensors for Ion and Molecule Recognition: The Next Chapter
}

\author{
Kai-Cheng Yan ${ }^{1}$, Axel Steinbrueck ${ }^{2}$, Adam C. Sedgwick ${ }^{2 *}$ and Tony D. James ${ }^{1,3 *}$ \\ ${ }^{1}$ Department of Chemistry, University of Bath, Bath, United Kingdom, ${ }^{2}$ Department of Chemistry, The University of Texas at \\ Austin, Austin, TX, United States, ${ }^{3}$ School of Chemistry and Chemical Engineering, Henan Normal University, Xinxiang, China
}

Over the past 30 years fluorescent chemosensors have evolved to incorporate many optical-based modalities and strategies. In this perspective we seek to highlight the current state of the art as well as provide our viewpoint on the most significant future challenges remaining in the area. To underscore current trends in the field and to facilitate understanding of the area, we provide the reader with appropriate contemporary examples. We then conclude with our thoughts on the most probable directions that chemosensor development will take in the not-too-distant future.

\section{OPEN ACCESS}

Edited by:

Dermot Diamond,

Dublin City University, Ireland

Reviewed by:

Ashok Kumar,

VIT University, India

Ananta Kumar Atta,

National Institute of Technology,

Arunachal Pradesh, India

*Correspondence:

Adam C. Sedgwick

a.c.sedgwick@utexas.edu

Tony D. James

t.d.james@bath.ac.uk.

Specialty section:

This article was submitted to

Chemical Sensors,

a section of the journal

Frontiers in Sensors

Received: 28 June 2021

Accepted: 19 August 2021

Published: 16 September 2021

Citation:

Yan K-C, Steinbrueck A, Sedgwick AC and James TD (2021) Fluorescent

Chemosensors for lon and Molecule

Recognition: The Next Chapter.

Front. Sens. 2:731928.

doi: 10.3389/fsens.2021.731928
Keywords: fluorescence, chemosensor, sensing, imaging, diagnostics, therapy

\section{INTRODUCTION}

In 1993 Anthony W. Czarnik published a seminal book on "Fluorescent Chemosensors for Ion and Molecule Recognition". (Czarnik, 1993). The book was a collection of chapters prepared by leaders in the area of Chemosensor research and provided insight into their research as well as their vision for the future directions of the field. Since that treatise was published, the area of molecular sensing has grown exponentially and the field has expanded to incorporate various other optical-based modalities and strategies e.g., chemiluminescence, photoacoustic/photothermal, and superresolution imaging. Here we pay homage to that trailblazing book and provide this perspective as an addendum (the next chapter), highlighting what has been achieved (current state of the art), what will be realized soon, and the future challenges (Wu et al., 2017; Tian X. et al., 2021). In doing so, we have judiciously selected key examples that are then discussed to enable the reader to gain an overview of the current state of play in the field. We then conclude this perspective with our thoughts on what directions we expect the development of chemosensors to take over the next several years.

A fluorescent chemosensor was originally defined as "a compound of abiotic origin that complexes to an analyte reversibly with concomitant fluorescence signal transduction" (Czarnik, 1993). This term is now used interchangeably with the term "fluorescent probe". However, for general understanding, a probe/chemosensor is defined as any compound that incorporates a binding/reactive site and a luminophore, as well as a mechanism for communication between these two sites. This ensures a measurable output is observed upon the probe's interaction with the target analyte. During the early years, one of the first major hurdles encountered for fluorescent chemosensors was the design of suitable receptors (binding-based units) and locking groups (reaction-based units) (Wu et al., 2019; Chang et al., 2020) that would allow for the selective targeting of a specific species/analyte within a particular sensing environment (e.g., aqueous media). Within the initial reports, poor selectivity was commonly observed and the reported chemosensors were consequently often considered as "proof of concept". However, these reports inspired researchers to join the effort to develop fluorescent chemosensors for a wide variety of 
<smiles>CC1(C)OB(c2ccc3c(c2)Oc2cc(OS(=O)(=O)c4ccc([N+](=O)[O-])cc4[N+](=O)[O-])ccc2C32OC(=O)c3ccccc32)OC1(C)C</smiles>

1<smiles>[O-]C1=C(O)C=C(/C=C/C2=C(Cl)/C(=C/C=C3\C=C(c4ccccc4)Oc4c3cc3c5c4CCCN5CCC3)CCC2)c2cc3c4c(c2O1)CCCN4CCC3</smiles>

2<smiles>CCOC(=O)c1c2ccc(B3OC(C)(C)C(C)(C)O3)ccc-2c(C(=O)OCC)c1N</smiles>

3

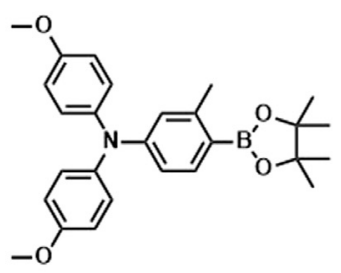

$6 a$

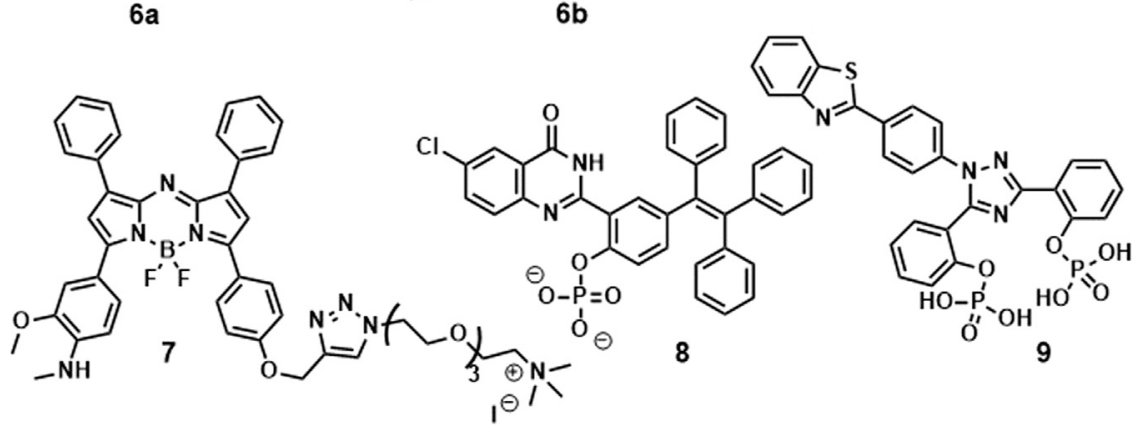<smiles>COC1(c2cc(B3OC(C)(C)C(C)(C)O3)c(/C=C/C(=O)O)cc2/C=C/C2=CC(=C(C#N)C#N)c3ccccc3O2)C(C)(C)OOC12C1CC3CC(C1)CC2C3</smiles><smiles>COC1(c2cc(OC3OC(CO)C(O)C(O)[C@H]3O)ccc2/C=C/C2=CC(=C(C#N)C#N)c3ccccc3O2)CC2CC3CC2CC1C3</smiles>

5

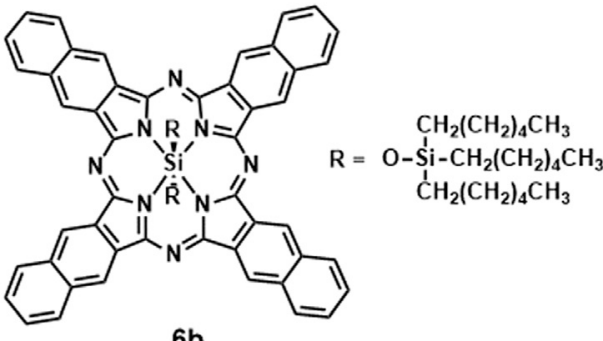

$6 b$

FIGURE 1 | Fluorescent chemosensors 1-9.

interdisciplinary and practical applications, of which we will now briefly highlight some of the most important trends. For detailed discussions on fluorescent chemosensor design and potential applications, we direct the reader to several excellent reviews (Wu et al., 2017; Sedgwick et al., 2018b; Wu et al., 2020; Feng et al., 2021; Han et al., 2021; Ma et al., 2021; Sedgwick et al., 2021a; Tian H. et al., 2021; Wu et al., 2021b).

Currently, researchers have access to a plethora of fluorescent chemosensors that allow for the detection of almost any diseasebased biomarker in vitro and in vivo. These chemosensors are regarded as chemical tools that can be employed to enhance our understanding of various pathological processes through the targeted visualization of key steps and biological intermediates (Han et al., 2021). Moreover, several technological advancements have led to the incorporation of fluorescent chemosensors into various sensor technologies that can provide meaningful qualityof-life improvements for consumers. This includes the use of a fluorescent chemosensor as part of the Eversense device for the real-time monitoring of blood glucose concentrations in diabetes sufferers (Mortellaro and DeHennis, 2014).

Fluorescent chemosensors have already shown great promise in real-world situations and applications, including but not limited to: the detection of environmental waste pollutants, detection of chemical warfare agents, fluorescence guided surgery, whole body diagnostic imaging, in vivo flow cytometry, and eHealth diagnostic devices (point of care (POC) testing) (Christodouleas et al., 2018; Jiao et al., 2020; Wei and Xunbin, 2021). In addition, colorimetric sensors have been established as potentially attractive sensing platforms due to their low cost as well as their capability to respond to stimuli with concurrent colour (UV-Vis) changes that could be discerned using the naked eye (López-Alled et al., 2017; Gwynne et al., 2021a; Gwynne et al., 2021b). This means colorimetric sensors are often seen as ideal diagnostic systems for non-expert users (Liu B. et al., 2020). However, in the context of this perspective, we have solely focused on fluorescent chemosensors. Nonetheless, 
<smiles></smiles>

10

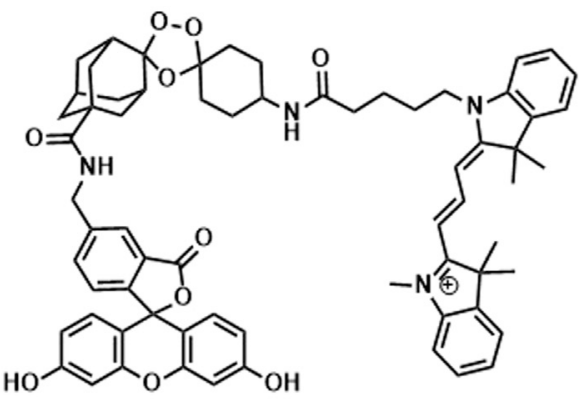

11<smiles>CN(CCn1cc(COC2OC(CO)[C@@H](O)[C@H](O)[C@H]2O)nn1)c1ccc(/C=C/C2=CC(=C(C#N)C#N)C=C(C(C)(C)C)O2)cc1</smiles>

12

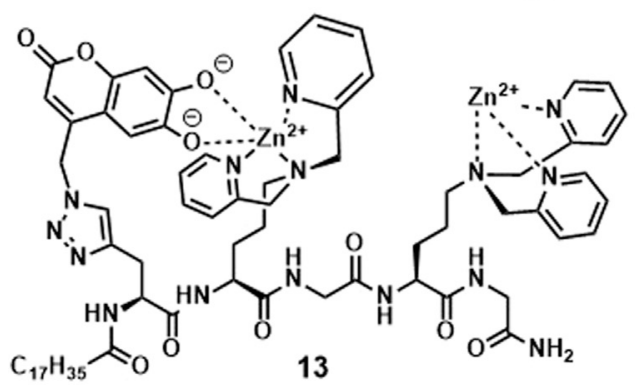<smiles>CC(=O)Oc1ccc(CN2c3ccc(B4OC(C)(C)C(C)(C)O4)cc3Oc3cc(B4OC(C)(C)C(C)(C)O4)ccc32)cc1</smiles>

14

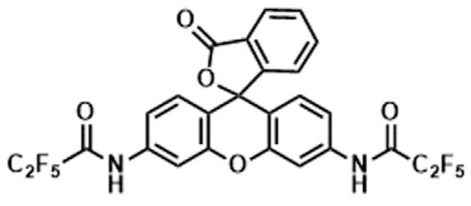

15<smiles>CN(C)c1ccc2c3c(cccc13)C(=O)N(CCNC(=O)CC[N+]1=C(/C=C/c3cc([N+](=O)[O-])ccc3OC3OC(CO)C(O)[C@H](O)[C@H]3O)C(C)(C)c3ccccc31)C2=O</smiles>

FIGURE 2 | Fluorescent chemosensors 10-16.

fluorescent systems often generate a colorimetric response in addition to a fluorescent output. This is due to the fact that a change in UV absorption (colour) is often coincidental with a change in fluorescence emission for any given chemosensor (Gwynne et al., 2019). Despite these advances, significant improvements to existing technologies needs to be made in order to address the current challenges in the field; these include: facilitating the generation of a measurable signal response by improving the signal to noise ratio, increasing the penetration depth of light to facilitate reliable detection of analytes within biological tissues or solid materials, the tuning of the output emission wavelength, as well as modulating the chemosensor solubility to provide optimized compatibility under various applications, and increasing analyte sensitivity and selectivity to improve the precision of chemosensors for any desired target. Below we discuss the current state of the art and the various strategies that are being developed to address the current limitations of modern sensing technologies.

\section{CURRENT STATE OF THE ART}

Traditionally, efforts to improve analyte selectivity have focused on the synthetic modification of known receptor units. However, at this moment in time, alternative approaches towards achieving target specificity have garnered increasing interest. This in part has been driven by the existence of an extensive library of off the shelf receptor units which has blunted the need to establish 
bespoke receptors with enhanced selectivity via synthetic optimization. Consequently, a particularly effective strategy using dual-locked probes (molecular logic gates) has recently emerged (Kolanowski et al., 2018; Wu et al., 2021a). Dual-locked systems are particularly attractive as they provide improved selectivity for the targeted system since they are only activated when at least two or more biomarkers that are associated with the targeted disease are present.

A particularly illustrative example of a dual locked system is our reported probe GSH-PF3 (1) (Sedgwick et al., 2018a; Figure 1). This system was designed to activate only when both the reactive oxygen species (ROS) (Wu et al., 2019; Yan et al., 2019) peroxynitrite and an important biological antioxidant glutathione (GSH) were present. The underlying design concept exploits the fact that GSH and other biological thiols play key roles in maintaining intracellular redox homeostasis, a process that is often altered within pathological settings (Kolanowski et al., 2018). Probe 1 demonstrated the ability to monitor the redox state of an appropriate cellular model and proved capable of visualizing the effect of a given therapeutic on the cellular redox environment (Sedgwick et al., 2018a). We anticipate that probe designs similar to 1 will allow the screening of drug candidates for a desired therapeutic outcome and thereby facilitate the more rapid identification of promising lead drug candidates. Expanding on this given example, we envision that numerous dual-locked strategies will continue to emerge as selectivity issues can be overcome by adding more than one "selectivity filter" (locking groups) to well-known sensing systems. In addition, these systems have exhibited excellent capability to overcome problems that are commonly associated with the use of multiple independent fluorescent probes during the investigation of relationships between more than one biological species/ stimulus. This is of particular interest because the use of separate probes for each specific targeted stimulus can result in erroneous analytical data due to different permeability, distribution, and biocompatibility of each individual probe (Wu et al., 2021a).

The key challenge of reliably achieving a measurable output signal is particularly problematic for in vivo imaging due to high background noise and observed autofluorescence, poor penetration depths of light into tissue, as well as significant light scattering. Current strategies aimed at improving signal output include the development of NIR-II systems (absorb/emit: 1,000-1,350 nm), chemiluminescence, (Yang et al., 2020), afterglow luminescence, ( $\mathrm{Li}$ and $\mathrm{Pu}, 2019$ ), photoacoustic (PA), (Knox and Chan, 2018), and two photon (TP) methods (Wu et al., 2021b).

NIR-II fluorophores have attracted significant interest as they provide higher image resolution when compared to NIR-I dyes (700-950 nm) (Liu P. et al., 2020). As an example, the group of Sletten developed NIR-II flavylium polymethine dyes such as JuloFlav7 (2), which were demonstrated to achieve high resolution in animal and tissue-based imaging experiments (Cosco et al., 2020). Although significant promise has been observed for these systems as imaging agents, the poor solubility of these NIR-II systems often requires their incorporation into nanoparticle formulations. As such, further research into overcoming these solubility issues is highly desirable. We would like to emphasize that aqueous solubility is an important design criterion for molecular sensors. This is due to the occurrence of unknown toxicities associated with nanoparticle formulations (De Jong and Borm, 2008) or toxicities associated with the use of organic media for colorimetric systems. We anticipate that the number of watersoluble NIR-II platforms will continue to increase over the coming years. Towards this end, an important strategy to overcome common solubility issues of chemosensors is the introduction of hydrophilic saccharide moieties either as appended motifs to enhance water-solubility or as a locking group to confer additional sensing properties via interaction with the desired target (Cho and Jung, 2015; Gim et al., 2019; Hazarika et al., 2020; Thomas et al., 2020).

In light of the current lack of NIR-II systems, TP imaging provides the ability to excite common fluorescent platforms with a "NIR-II-like" excitation source. Due to this capability, TP is increasingly becoming ubiquitous, however, the development of TP systems is being slowed by the limited availability of suitable $\mathrm{TP}$ dyes and as a result there is a lack of appropriate small molecule-based fluorescent chemosensor systems currently available. A recent example of a novel TP system was reported by the group of Lewis using an azulene-based scaffold AzuFluor 483-Bpin (3). Significantly, these AzuFluors ${ }^{\circledR}$ present themselves as amenable core units for the development of TP probes (Murfin et al., 2019).

An attractive alternative for improving light penetration is the elimination of the requirement for a light excitation source by using chemiluminescent probes that emit light only when initiated by a suitable chemical reaction. In contrast to conventional fluorescence imaging, the removal of the excitation light source significantly minimizes the issues of background signals and thereby provides a high signal to noise ratio as was shown both in vitro and in vivo. Significant efforts from the Shabat group (Hananya and Shabat, 2017; Gnaim et al., 2018; Gnaim and Shabat, 2019) have demonstrated that the core dioxetane chemiluminescent structure can be modified to function with any appropriate locking group, with proven examples including boronate ester 4 for ROS detection and a galactose unit responsive to $\beta$-galactosidase 5 . Through their endeavours, an attractive NIR-chemiluminescent scaffold was developed. The reaction of NIR-chemiluminescent probes 4 and 5 with their respective targets led to the emission of NIR light that was shown suitable for in vivo imaging (Green et al., 2017).

The group of $\mathrm{Pu}$ have explored the use of organic-based afterglow imaging in an effort to enable improved animal and tissue imaging (Dang et al., 2020). Afterglow imaging provides enhanced sensitivity, when compared with traditional methods, as the long-lived emissive species is easily detectable over the comparatively short-lived background auto fluorescence from endogenous biological molecules. This can be seen as analogous to time-gated luminescence with the use of inorganic lanthanide systems (Hananya and Shabat, 2017; Gnaim et al., 2018; Gnaim and Shabat, 2019; Bodman and Butler, 2021). Many organic molecules with room-temperature 
phosphorescence (RTP) can be seen as suitable afterglow agents. However, most RTP luminogens only emit visible light, which provides shallow tissue penetration depth, thereby constraining possible in vivo applications. To overcome this limitation, $\mathrm{Pu}$ has developed an RTP nanoprobe with NIR emission for in vivo afterglow imaging. The nanoparticle-based probe was constructed using an amphiphilic triblock copolymer (F127), mTPA (6a) as the phosphorescence generator, and an NIRfluorescent dye NCBS (6b) as the energy acceptor providing redshifted phosphorescent emission at $780 \mathrm{~nm}$ (Dang et al., 2020). This phenomenon is known as room-temperature phosphorescence resonance energy transfer (RT-PRET). These redshifted afterglow luminescence probes exhibited minimal background interference and thereby enabled lymph node imaging of live mice. As such, these probes provide excellent insight as a starting point for the construction of other organicbased NIR afterglow imaging agents using RT-PRET.

Another approach capable of achieving enhanced tissue penetration depth is photoacoustic (PA) imaging, where optical excitation is coupled with acoustic detection (light in, sound out), thereby enabling high resolution and deep tissue imaging at centimetre depths (Knox and Chan, 2018; Cheng et al., 2020; Ren et al., 2020; Chen et al., 2021; Lucero et al., 2021). PA probes that can be activated by specific biological species are of particular interest as they have the potential to complement other clinically used diagnostic systems (i.e., Ultrasound) for disease diagnosis. Within this arena, the Chan group has developed nitric oxide (NO) triggered probes for acoustogenic imaging (Lucero et al., 2021). For example APNO-5 (7), could be used for the ratiometric imaging of endogenous $\mathrm{NO}$ in vivo (BALB/c mice) in a murine lipopolysaccharide-induced inflammation model (Reinhardt et al., 2018).

As seen with the above examples, the response of a chemosensor can be enhanced through the response mechanism. One area that has attracted significant interest over the past decades is Aggregation Induced Emission (AIE), a term which was coined in 2001 by the group of Tang (Luo et al., 2001). Unlike the majority of conventional dyes that undergo aggregation-caused quenching (ACQ), AIE luminogens (AIEgens) show weak or negligible emission in dilute solution but emit intensely in an aggregated state/under conditions of restricted motion (Song et al., 2020). Sensors based on AIE exhibit certain advantages over traditional fluorophores, in fact, the aggregative nature means that the sensor accumulates at a particular location and allows accurate positional biomarker detection. This is particularly relevant because commonly used fluorescent probes/chemosensors are subject to diffusion and can consequently be prone to provide false information on a biomarker's true location.

A noteworthy example is the AIE probe for the monitoring of alkaline phosphatase (ALP) (Sedgwick et al., 2018b) enzyme activity in cells developed by the Zhang group. The precipitation of the unmasked excited state intramolecular proton transfer (ESIPT)-fluorophore HTPQA (8) occurred upon exposure to ALP, which facilitated diffusion resistant monitoring of ALP activity in osteosarcoma cells and tissue (Liu et al., 2017). Another, ALP responsive AIE system,
ExPhos (9), was reported by the Sessler group (Sedgwick et al., 2021b). This system was based on deferasirox (ExJade) an FDAapproved iron chelator, which is used to treat patients with iron overload. Owing to the pathological role of iron, this system enabled the tracking and detection of disease-based biomarkers while providing inherent therapeutic properties (Steinbrueck et al., 2021; Steinbrueck et al., 2020).

Organic fluorophores with dual-emission and large Stokes shifts are desired for many applications including biological imaging. Within this arena, a new luminescence mechanism termed vibration-induced emission (VIE), which possess these desired properties, has recently been established. The VIE mechanism was coined because different emissions are observed from the planar (long-wavelength) and the bent forms (short-wavelength) of the V-shaped $N, N^{\prime}$-disubstitudeddihydribenzo [a,c]phenazines (DHPs) (Zhang et al., 2020).

The group of Tian developed probe VN (10) as a VIE system to detect amyloid $\beta(\mathrm{A} \beta)$ fibrils which are associated with the development of the neurodegenerative disorder Alzheimer's disease (AD) (Dou et al., 2017; Figure 2). In fact, probe 10 could be used to detect $A \beta$ in solution. In the absence of $A \beta$ fibrils 10 exhibited orange/red emission at $600 \mathrm{~nm}$, however, upon the addition of $\mathrm{A} \beta 42$ peptide, the blue emission at $470 \mathrm{~nm}$ increased. The selective enhancement of blue emission was attributed to binding of 10 with $A \beta 42$, limiting the vibration of the excited state. Significantly, 10 could be used to image $A \beta$ plaques and illustrates the potential of VIE based probes for biological imaging.

Another important approach for the improvement of the output profile of a chemosensor is the use of Förster resonance energy transfer (FRET). With FRET based systems, the apparent stokes shift of the chemosensor is extended through an energy transfer from the donor to the acceptor, resulting in fluorescence output from the acceptor at a longer wavelength (Wu et al., 2020). A representative FRET system FIP-1 (11) was reported by the Chang group. This iron responsive probe, featuring a 5-aminomethyl fluorescein (5-AMF) donor motif and a cyanine $3(\mathrm{Cy} 3)$ acceptor as the FRET pair, was used for visualising labile iron pools in living systems during ferroptosis therefore allowing for the monitoring of iron signalling in living systems (Aron et al., 2016).

Obviously, combining all these aspects into one molecular species can be a daunting task, that is why ensemble-based approaches towards such systems should be explored. This simple strategy requires the knowledge of host-guest chemistry, the hallmark of the field of supramolecular chemistry. The simplicity of combining molecular sensors with materials/host platforms improves their overall targeting ability for in vivo applications, minimizes synthetic effort, and provides a potential route to theranostic applications (incorporation of a therapeutic into the diagnostic supramolecular ensemble) ( $\mathrm{Hu}$ et al., 2020).

Extensive efforts from the group of $\mathrm{He}$ have led to the development of receptor-targeting fluorescence imaging and theranostic systems using graphene oxide (GO) based supramolecular glycocomposites. (Ji et al., 2015; Dou et al., 2021). Many disease-related processes involve glycoligand- 
receptor interactions. GO based supramolecular fluorescent glycocomposites are constructed using a hydrophobic fluorophore with linked glycoligands. Owing to their design, significantly enhanced cellular and tissue imaging ability of the fluorescent glycoprobe is observed due to glycoligand clustering enhancing interactions with the cell surface receptors. An example is DK1 (12) where GO enhanced clustering to strengthen the interactions between the cancer cell receptors and the glycoprobe (galactose) was observed (Ji et al., 2015). In addition, the composite could be further converted into a theranostic system by coassembling the glycoprobe and an anticancer drug onto the GO surface. As such, a theranostic supramolecular glycocomposite could be developed that was able to both image and kill liver cancer cells that overexpress the glycoprotein receptor.

To avoid extensive synthetic endeavours, the receptor and fluorophore do not have to be chemically linked since displacement-based systems remove such a need (Sedgwick et al., 2021a). A particularly interesting example of an intramolecular indicator displacement (IID) system for cell surface phosphatidylserine (PS) has been developed by the group of Jolliffe (Zwicker et al., 2019). With the system P-IID (13), a coumarin indicator is released from the intramolecularly linked receptor in the presence of cell surface PS, resulting in fluorescence "turn-on". Using this IID system in combination with confocal laser scanning microscopy allowed for the successful monitoring of a key cellular process that is activated during apoptosis in live cells.

As stated previously, the aqueous solubility of imaging systems is often a key challenge and this issue is seen frequently with cyanine and porphyrin-based systems, which are prone to undergo aggregation (Pasternack et al., 2000). As such, nanoparticle formulation is an essential approach to enhance solubility. Within this field, protein-based hybridization is an appealing strategy that exhibits ideal biocompatibility. A prominent example of this approach exploits the host-guest chemistry of human serum albumin (HSA) with highly lipophilic molecules such as cell impermeable and insoluble fluorescent probes (Sheng et al., 2014; Han et al., 2020; Jia et al., 2020; Sen et al., 2020; Sen et al., 2021).

The Pinkment-OAc (14)/HSA supramolecular hybrid was used in vitro to detect peroxynitrite $\left(\mathrm{ONOO}^{-}\right)$with enhanced response (Han et al., 2020). Hybrid 14/HSA was used to monitor $\mathrm{ONOO}^{-}$in RAW 264.7 macrophages and HeLa cancer cells. Importantly, the prepared hybrid exhibited good cell permeability and facilitated the detection of $\mathrm{ONOO}^{-}$in vivo in mice. Pinkment-OAc alone was shown to be ineffective for both cellular and in vivo imaging, which cemented the significance of protein-based hybridization as a strategy for the enhancement of biocompatibility.

In a separate study, this strategy was used as an effective means to detect enzyme activity. ACS-HNE (15) was used for the selective detection of neutrophil elastase (NE) in RAW 264.7 cells (Jia et al., 2020). Probe 15 exhibited excellent selectivity toward elastase over other biological analytes and enzymes. Using this approach, limitations that arose from the comparatively poor solubility and limited cell permeability of 15 alone could be overcome using the 15/HSA supramolecular hybrid.
Super-resolution-based technologies have revolutionized fluorescence imaging by circumventing the diffraction limit of light and thereby enabling visualization of structures with nanoscale accuracy within a cellular environment (Carvalhais et al., 2021). Amongst the super-resolution techniques available we would like to specifically highlight Stochastic Optical Reconstruction Microscopy (STORM) (Rust et al., 2006; Samanta et al., 2019) and photoactivated localization microscopy (PALM) (Betzig et al., 2006) as these techniques use probes with switchable fluorescence emission profiles (photoblinking).

$\mathrm{He}$ and co-workers developed a $\beta$-galactosidase ( $\beta$-Gal)responsive photochromic fluorophore $\mathrm{NpG}$ (16), that was able to form an HSA supramolecular hybrid (Chai et al., 2020).16@ HSA hybrid formation enhanced the fluorescence emission $(520 \mathrm{~nm})$ and facilitated the visualization of the spiropyran's fluorescence output in aqueous media. Subsequent cleavage of the galactose unit by $\beta$-Gal of the 16@HSA hybrid resulted in the formation of NpM@HSA, generating an enhanced red fluorescence emission $(620 \mathrm{~nm})$. The formed merocyanine unit experiences photoisomerization (merocyanine $\leftrightarrow$ spiropyran), thereby enabling STORM imaging while displaying excellent photostability/reversibility with minimal phototoxicity. Using STORM and 16@HSA, the subcellular distribution of $\beta$-Gal activity between different cell lines could be determined with nanoscale precision.

Given the success of the supramolecular hybrid strategy using HSA with these probes, we anticipate that HSA/fluorescent probe hybrids are one of the best methods to overcome solubility issues associated with hydrophobic fluorescent imaging agents that are designed to detect disease related biomarkers and as such we anticipate that this simple supramolecular approach will be widely adopted by the sensing and imaging community.

\section{WHERE NEXT?}

Due to the continuous need for new and effective therapeutics, the development of chemical tools that can be used to evaluate various biological pathways in vitro and in vivo is more important than ever. Efforts in the sensing community will continue to focus on the development of chemosensors in the endeavour to uncover new biological mechanisms and to identify the role of various biological species. Such chemosensor-based toolkits are crucial to illuminate the origins and progression of disease states. While the development of completely new receptor motifs is rare, we anticipate that fluorescent chemosensors that incorporate novel receptor units will continue to emerge for the investigation of fresh biological targets. One such an example is the development of a thioredoxin reductase selective fluorescent probe developed by Fang and co-workers (Zhang et al., 2014). Significantly, this biomarker is a key target for gold (Au)-based chemotherapeutics and it is anticipated that this tool could be crucial in assisting the development of these attractive drug candidates for clinical use (Yeo et al., 2018). Over the coming years, we expect that similar examples of chemical tools for new biological targets will continue to be developed. However, the 
archetypal challenges associated with chemosensor development will need to be successfully addressed in parallel during their development. Those challenges include but are not limited to: aqueous solubility, analyte selectivity, biocompatibility, and laborious time-intensive synthetic optimization.

Due to the complexity of cellular processes, we anticipate that dual-locked systems will continue to be developed and emerge in the literature. This is because such tools can help to improve the fundamental understanding of key biological pathways or help to identify currently unknown relationships between biomarkers that contribute to a specific disease state. Owing to the high signal-to-noise ratio of chemiluminescent probes, we are particularly hopeful that such systems will continue to be developed to help shed light on these difficult problems. Currently, chemiluminescence is a relatively unexplored area; however, the seminal research by the Shabat group has inspired other groups to focus on these potentially game changing systems (Hananya et al., 2016). As a result, we anticipate that new chemiluminescence scaffolds and strategies will continue to emerge over the next several years. However, as with fluorescence-based systems, the major challenge remains the development of aqueous soluble and biocompatible systems.

With regards to in vivo applications, we anticipate that NIR-II chemosensors will continue to be developed owing to their enhanced tissue penetration and higher image resolution with respect to systems that depend on visible light (Li et al., 2020). Owing to the "light-in, sound out" approach of PA imaging, we anticipate that PA based chemosensors will remain one of the "hottest" topics in the chemosensor area and will continue to evolve as PA technology improves. The strong interest in PA systems is driven by the potential clinical utility of such PA systems (Attia et al., 2019). Again, the major challenge to PA chemosensor development is the design of systems that avoid both synthetic and solubility issues. Due to the use of a pulsed laser, photostability of current PA systems is a common problem. We have confidence that if current challenges and limitations of NIR-II systems can be overcome, unprecedented opportunities for the investigation of biological process in vivo will be possible that have thus far only been realized in vitro. Such advancements would allow researchers to solve unknown biological problems and thereby unlock stimulating new biological questions. Moreover, with the recent developments in the area of personalised medicine, (Goetz and Schork, 2018), we anticipate that NIR-II systems will enable a better understanding and hence an improved ability to adjust to differences between individual patients during specific treatment plans. As we write this perspective, very few NIR-II fluorescent and PA examples exist, which we presume to be the result of synthetic challenges coupled with known solubility issues. Therefore, both NIR-II and PA based systems represent a new Frontier for development that is waiting to be explored by a new generation of chemosensor pioneers.

\section{REFERENCES}

Aron, A. T., Loehr, M. O., Bogena, J., and Chang, C. J. (2016). An Endoperoxide Reactivity-Based FRET Probe for Ratiometric Fluorescence Imaging of Labile
Within this perspective we have on the whole limited our discussion to systems that selectively detect biological analytes and function as chemosensors, however, many systems that interact with light can also be used for photodynamic therapy (PDT) and photothermal therapy (PTT). These systems are often plagued with off-target toxicities or result in undefined/ large tumour margins resulting in unwanted phototoxicities. In recent years, efforts from several research groups have focused on the development of combined and activatable chemosensors with PDT/PTT properties to overcome these challenges. A particularly nice example is the aminopeptidase $\mathrm{N}$ responsive PDT agent by Peng and co-workers, which enables the simultaneous detection of APN in tumors and subsequent treatment (Zhou et al., 2020). Given that this approach is still embryonic in nature, we expect that numerous examples will continue to emerge as the area develops.

With reference to the original Czarnik collection, the field of chemosensors has evolved considerably over the past 30 years, and we foresee that the area will continue to develop to meet the everchanging medicinal needs of individuals over the next 30 years. While it is impossible to predict what will emerge and develop in the future, we are convinced that the field of chemosensors will continue to grow and inspire new generations of researchers who will contribute towards the ongoing efforts and provide fresh and ingenious solutions to the current bottlenecks in chemosensor synthesis, biocompatibility, and solubility.

\section{DATA AVAILABILITY STATEMENT}

The original contributions presented in the study are included in the article/Supplementary Material, further inquiries can be directed to the corresponding authors.

\section{AUTHOR CONTRIBUTIONS}

TDJ and ACS conceived the perspective and K-CY and AS provided support with referencing and figure preparation.

\section{ACKNOWLEDGMENTS}

TDJ wishes to thank the Royal Society for a Wolfson Research Merit Award and the Open Research Fund of the School of Chemistry and Chemical Engineering, Henan Normal University for support (2020ZD01). TDJ, ACS and K-CY would like to thank the University of Bath for support. ACS and AS would like to thank The University of Texas at Austin for support.

Iron Pools in Living Cells. J. Am. Chem. Soc. 138, 14338-14346. doi:10.1021/ jacs.6b08016

Attia, A. B. E., Balasundaram, G., Moothanchery, M., Dinish, U. S., Bi, R., Ntziachristos, V., et al. (2019). A Review of Clinical Photoacoustic Imaging: Current and Future Trends. Photoacoustics 16, 100144. doi:10.1016/j.pacs.2019.100144 
Betzig, E., Patterson, G. H., Sougrat, R., Lindwasser, O. W., Olenych, S., Bonifacino, J. S., et al. (2006). Imaging Intracellular Fluorescent Proteins at Nanometer Resolution. Science 313, 1642-1645. doi:10.1126/science.1127344

Bodman, S. E., and Butler, S. J. (2021). Advances in Anion Binding and Sensing Using Luminescent Lanthanide Complexes. Chem. Sci. 12, 2716-2734. doi:10.1039/d0sc05419d

Carvalhais, L. G., Martinho, V. C., Ferreiro, E., and Pinheiro, P. S. (2021). Unraveling the Nanoscopic Organization and Function of Central Mammalian Presynapses With Super-Resolution Microscopy. Front. Neurosci. 14, 1379. doi:10.3389/fnins.2020.578409

Chai, X., Han, H.-H., Sedgwick, A. C., Li, N., Zang, Y., James, T. D., et al. (2020). Photochromic Fluorescent Probe Strategy for the Super-resolution Imaging of Biologically Important Biomarkers. J. Am. Chem. Soc. 142, 18005-18013. doi:10.1021/jacs.0c05379

Chang, C. J., James, T. D., New, E. J., and Tang, B. Z. (2020). Activity-Based Sensing: Achieving Chemical Selectivity through Chemical Reactivity. Acc. Chem. Res. 53, 1. doi:10.1021/acs.accounts.9b00542

Chen, J., Sedgwick, A. C., Sen, S., Ren, Y., Sun, Q., Chau, C., et al. (2021). Expanded Porphyrins: Functional Photoacoustic Imaging Agents that Operate in the NIRII Region. Chem. Sci. 12, 9916-9921. doi:10.1039/d1sc01591e

Cheng, H.-B., Li, Y., Tang, B. Z., and Yoon, J. (2020). Assembly Strategies of OrganicBased Imaging Agents for Fluorescence and Photoacoustic Bioimaging Applications. Chem. Soc. Rev. 49, 21-31. doi:10.1039/c9cs00326f

Cho, E., and Jung, S. (2015). Supramolecular Complexation of Carbohydrates for the Bioavailability Enhancement of Poorly Soluble Drugs. Molecules 20, 19620-19646. doi:10.3390/molecules201019620

Christodouleas, D. C., Kaur, B., and Chorti, P. (2018). From Point-of-Care Testing to eHealth Diagnostic Devices (eDiagnostics). ACS Cent. Sci. 4, 1600-1616. doi:10.1021/acscentsci.8b00625

Cosco, E. D., Spearman, A. L., Ramakrishnan, S., Lingg, J. G. P., Saccomano, M., Pengshung, M., et al. (2020). Shortwave Infrared Polymethine Fluorophores Matched to Excitation Lasers Enable Non-invasive, Multicolour In Vivo Imaging in Real Time. Nat. Chem. 12, 1123-1130. doi:10.1038/s41557-020-00554-5

Czarnik, A. W. (1993). Fluorescent Chemosensors for Ion and Molecule Recognition. Washington, D.C.: American Chemical Society.

Dang, Q., Jiang, Y., Wang, J., Wang, J., Zhang, Q., Zhang, M., et al. (2020). RoomTemperature Phosphorescence Resonance Energy Transfer for Construction of Near-Infrared Afterglow Imaging Agents. Adv. Mater. 32, 2006752. doi:10.1002/adma.202006752

De Jong, W. H., and Borm, P. J. A. (2008). Drug Delivery and Nanoparticles: Applications and Hazards. Int. J. Nanomed. 3, 133-149. doi:10.2147/ijn.s596

Dou, W.-T., Chen, W., He, X.-P., Su, J., and Tian, H. (2017). Vibration-InducedEmission (VIE) for Imaging Amyloid $\beta$ Fibrils. Faraday Discuss. 196, 395-402. doi:10.1039/c6fd00156d

Dou, W.-T., Xu, F., Xu, C.-X., Gao, J., Ru, H.-B., Luan, X., et al. (2021). Graphene Nanoribbon-Based Supramolecular Ensembles with Dual-Receptor Targeting Function for Targeted Photothermal Tumor Therapy. Chem. Sci. 12, 11089-11097. doi:10.1039/d1sc02154k

Feng, L., Ning, J., Tian, X., Wang, C., Yu, Z., Huo, X., et al. (2021). Fluorescent Probes for the Detection and Imaging of Cytochrome P450. Coord. Chem. Rev. 437, 213740. doi:10.1016/j.ccr.2020.213740

Gim, S., Zhu, Y., Seeberger, P. H., and Delbianco, M. (2019). CarbohydrateBased Nanomaterials for Biomedical Applications. WIREs Nanomed. Nanobi. 11, e1558. doi:10.1002/wnan.1558

Gnaim, S., Green, O., and Shabat, D. (2018). The Emergence of Aqueous Chemiluminescence: New Promising Class of Phenoxy 1,2-dioxetane Luminophores. Chem. Commun. 54, 2073-2085. doi:10.1039/c8cc00428e

Gnaim, S., and Shabat, D. (2019). Activity-Based Optical Sensing Enabled by SelfImmolative Scaffolds: Monitoring of Release Events by Fluorescence or Chemiluminescence Output. Acc. Chem. Res. 52, 2806-2817. doi:10.1021/ acs.accounts. 9 b00338

Goetz, L. H., and Schork, N. J. (2018). Personalized Medicine: Motivation, Challenges, and Progress. Fertil. Sterility 109, 952-963. doi:10.1016/j.fertnstert.2018.05.006

Green, O., Gnaim, S., Blau, R., Eldar-Boock, A., Satchi-Fainaro, R., and Shabat, D. (2017). Near-Infrared Dioxetane Luminophores with Direct Chemiluminescence Emission Mode. J. Am. Chem. Soc. 139, 13243-13248. doi:10.1021/jacs.7b08446

Gwynne, L., Sedgwick, A. C., Gardiner, J. E., Williams, G. T., Kim, G., Lowe, J. P., et al. (2019). Long Wavelength TCF-Based Fluorescent Probe for the Detection of Alkaline Phosphatase in Live Cells. Front. Chem. 7, 255. doi:10.3389/ fchem.2019.00255

Gwynne, L., Williams, G. T., Yan, K.-C., Patenall, B. L., Gardiner, J. E., He, X.-P., et al. (2021b). TCF-ALP: a Fluorescent Probe for the Selective Detection of Staphylococcus Bacteria and Application in "smart" Wound Dressings. Biomater. Sci. 9, 4433-4439. doi:10.1039/d0bm01918f

Gwynne, L., Williams, G. T., Yan, K.-C., Gardiner, J. E., Hilton, K. L. F., Patenall, B. L., et al. (2021a). The Evaluation of Ester Functionalised TCF-Based Fluorescent Probes for the Detection of Bacterial Species. Isr. J. Chem. 61, 234-238. doi:10.1002/ijch.202000105

Han, H.-H., Sedgwick, A. C., Shang, Y., Li, N., Liu, T., Li, B.-H., et al. (2020). Protein Encapsulation: a New Approach for Improving the Capability of Small-Molecule Fluorogenic Probes. Chem. Sci. 11, 1107-1113. doi:10.1039/c9sc03961a

Han, H.-H., Tian, H., Zang, Y., Sedgwick, A. C., Li, J., Sessler, J. L., et al. (2021). Small-molecule Fluorescence-Based Probes for Interrogating Major Organ Diseases. Chem. Soc. Rev. doi:10.1039/D0CS01183E

Hananya, N., Eldar Boock, A., Bauer, C. R., Satchi-Fainaro, R., and Shabat, D. (2016). Remarkable Enhancement of Chemiluminescent Signal by DioxetaneFluorophore Conjugates: Turn-ON Chemiluminescence Probes with Color Modulation for Sensing and Imaging. J. Am. Chem. Soc. 138, 13438-13446. doi:10.1021/jacs.6b09173

Hananya, N., and Shabat, D. (2017). A Glowing Trajectory between Bio- and Chemiluminescence: From Luciferin-Based Probes to Triggerable Dioxetanes. Angew. Chem. Int. Ed. 56, 16454-16463. doi:10.1002/anie.201706969

Hazarika, S. I., Dolai, B., and Atta, A. K. (2020). Water Compatible Triazole Linked Pyrene-C1-Glucosyl Fluorescent Sensor for $\mathrm{Au}^{3+}$ and Living Cell Imaging Studies. J. Mol. Struc. 1202, 127272. doi:10.1016/j.molstruc.2019.127272

Hu, X.-L., Kwon, N., Yan, K.-C., Sedgwick, A. C., Chen, G.-R., He, X. P., et al. (2020). Bio-Conjugated Advanced Materials for Targeted Disease Theranostics. Adv. Funct. Mater. 30, 1907906. doi:10.1002/adfm.201907906

Ji, D.-K., Zhang, Y., Zang, Y., Liu, W., Zhang, X., Li, J., et al. (2015). Receptortargeting Fluorescence Imaging and Theranostics Using a Graphene Oxide Based Supramolecular Glycocomposite. J. Mater. Chem. B 3, 9182-9185. doi:10.1039/c5tb02057c

Jia, Z., Han, H.-H., Sedgwick, A. C., Williams, G. T., Gwynne, L., Brewster, J. T., et al. (2020). Protein Encapsulation: A Nanocarrier Approach to the Fluorescence Imaging of an Enzyme-Based Biomarker. Front. Chem. 8, 389. doi: $10.3389 /$ fchem. 2020.00389

Jiao, J., Zhang, J., Yang, F., Song, W., Han, D., Wen, W., et al. (2020). Quicker, Deeper and Stronger Imaging: A Review of Tumor-Targeted, Near-Infrared Fluorescent Dyes for Fluorescence Guided Surgery in the Preclinical and Clinical Stages. Eur. J. Pharm. Biopharm. 152, 123-143. doi:10.1016/j.ejpb.2020.05.002

Knox, H. J., and Chan, J. (2018). Acoustogenic Probes: A New Frontier in Photoacoustic Imaging. Acc. Chem. Res. 51, 2897-2905. doi:10.1021/ acs.accounts.8b00351

Kolanowski, J. L., Liu, F., and New, E. J. (2018). Fluorescent Probes for the Simultaneous Detection of Multiple Analytes in Biology. Chem. Soc. Rev. 47, 195-208. doi:10.1039/c7cs00528h

Li, B., Zhao, M., and Zhang, F. (2020). Rational Design of Near-Infrared-II Organic Molecular Dyes for Bioimaging and Biosensing. ACS Mater. Lett. 2, 905-917. doi:10.1021/acsmaterialslett.0c00157

Li, J., and Pu, K. (2019). Development of Organic Semiconducting Materials for Deep-Tissue Optical Imaging, Phototherapy and Photoactivation. Chem. Soc. Rev. 48, 38-71. doi:10.1039/c8cs00001h

Liu, B., Zhuang, J., and Wei, G. (2020a). Recent Advances in the Design of Colorimetric Sensors for Environmental Monitoring. Environ. Sci. Nano 7, 2195-2213. doi:10.1039/d0en00449a

Liu, H.-W., Li, K., Hu, X.-X., Zhu, L., Rong, Q., Liu, Y., et al. (2017). In Situ Localization of Enzyme Activity in Live Cells by a Molecular Probe Releasing a Precipitating Fluorochrome. Angew. Chem. Int. Ed. 56, 11788-11792. doi:10.1002/anie.201705747

Liu, P., Mu, X., Zhang, X.-D., and Ming, D. (2020b). The Near-Infrared-II Fluorophores and Advanced Microscopy Technologies Development and Application in Bioimaging. Bioconjug. Chem. 31, 260-275. doi:10.1021/acs.bioconjchem.9b00610

López-Alled, C. M., Sanchez-Fernandez, A., Edler, K. J., Sedgwick, A. C., Bull, S. D., McMullin, C. L., et al. (2017). Azulene-boronate Esters: Colorimetric Indicators for Fluoride in Drinking Water. Chem. Commun. 53, 12580-12583. doi:10.1039/c7cc07416f 
Lucero, M. Y., East, A. K., Reinhardt, C. J., Sedgwick, A. C., Su, S., Lee, M. C., et al. (2021). Development of NIR-II Photoacoustic Probes Tailored for Deep-Tissue Sensing of Nitric Oxide. J. Am. Chem. Soc. 143, 7196-7202. doi:10.1021/jacs.1c03004

Luo, J., Xie, Z., Lam, J. W. Y., Cheng, L., Chen, H., Qiu, C., et al. (2001). Aggregation-induced Emission of 1-Methyl-1,2,3,4,5-Pentaphenylsilole. Chem. Commun., 1740-1741. doi:10.1039/b105159h

Ma, S., Chen, G., Xu, J., Liu, Y., Li, G., Chen, T., et al. (2021). Current Strategies for the Development of Fluorescence-Based Molecular Probes for Visualizing the Enzymes and Proteins Associated with Alzheimer's Disease. Coord. Chem. Rev. 427, 213553. doi:10.1016/j.ccr.2020.213553

Mortellaro, M., and DeHennis, A. (2014). Performance Characterization of an Abiotic and Fluorescent-Based Continuous Glucose Monitoring System in Patients with Type 1 Diabetes. Biosens. Bioelectron. 61, 227-231. doi:10.1016/j.bios.2014.05.022

Murfin, L. C., Weber, M., Park, S. J., Kim, W. T., Lopez-Alled, C. M., McMullin, C. L., et al. (2019). Azulene-Derived Fluorescent Probe for Bioimaging: Detection of Reactive Oxygen and Nitrogen Species by Two-Photon Microscopy. J. Am. Chem. Soc. 141, 19389-19396. doi:10.1021/jacs.9b09813

Pasternack, R. F., Fleming, C., Herring, S., Collings, P. J., dePaula, J., DeCastro, G., et al. (2000). Aggregation Kinetics of Extended Porphyrin and Cyanine Dye Assemblies. Biophysical J. 79, 550-560. doi:10.1016/s0006-3495(00)76316-8

Reinhardt, C. J., Zhou, E. Y., Jorgensen, M. D., Partipilo, G., and Chan, J. (2018). A Ratiometric Acoustogenic Probe for In Vivo Imaging of Endogenous Nitric Oxide. J. Am. Chem. Soc. 140, 1011-1018. doi:10.1021/jacs.7b10783

Ren, Y., Sedgwick, A. C., Chen, J., Thiabaud, G., Chau, C. V., An, J., et al. (2020). Manganese(II) Texaphyrin: A Paramagnetic Photoacoustic Contrast Agent Activated by Near-IR Light. J. Am. Chem. Soc. 142, 16156-16160. doi:10.1021/jacs.0c04387

Rust, M. J., Bates, M., and Zhuang, X. (2006). Sub-diffraction-limit Imaging by Stochastic Optical Reconstruction Microscopy (STORM). Nat. Methods 3, 793-796. doi:10.1038/nmeth929

Samanta, S., Gong, W., Li, W., Sharma, A., Shim, I., Zhang, W., et al. (2019). Organic Fluorescent Probes for Stochastic Optical Reconstruction Microscopy (STORM): Recent Highlights and Future Possibilities. Coord. Chem. Rev. 380, 17-34. doi:10.1016/j.ccr.2018.08.006

Sedgwick, A. C., Brewster, J. T., Wu, T., Feng, X., Bull, S. D., Qian, X., et al. (2021a). Indicator Displacement Assays (IDAs): the Past, Present and Future. Chem. Soc. Rev. 50, 9-38. doi:10.1039/c9cs00538b

Sedgwick, A. C., Han, H.-H., Gardiner, J. E., Bull, S. D., He, X.-P., and James, T. D. (2018a). The Development of a Novel AND Logic Based Fluorescence Probe for the Detection of Peroxynitrite and GSH. Chem. Sci. 9, 3672-3676. doi:10.1039/c8sc00733k

Sedgwick, A. C., Wu, L., Han, H.-H., Bull, S. D., He, X.-P., James, T. D., et al. (2018b). Excited-state Intramolecular Proton-Transfer (ESIPT) Based Fluorescence Sensors and Imaging Agents. Chem. Soc. Rev. 47, 8842-8880. doi:10.1039/c8cs00185e

Sedgwick, A. C., Yan, K.-C., Mangel, D. N., Shang, Y., Steinbrueck, A., Han, H.-H., et al. (2021b). Deferasirox (ExJade): An FDA-Approved AIEgen Platform with Unique Photophysical Properties. J. Am. Chem. Soc. 143, 1278-1283. doi:10.1021/jacs.0c11641

Sen, S., Perrin, M. W., Sedgwick, A. C., Dunsky, E. Y., Lynch, V. M., He, X.-P., et al. (2020). Toward Multifunctional Anticancer Therapeutics: post-synthetic Carbonate Functionalisation of Asymmetric Au(i) Bis-N-Heterocyclic Carbenes. Chem. Commun. 56, 7877-7880. doi:10.1039/d0cc03339a

Sen, S., Perrin, M. W., Sedgwick, A. C., Lynch, V. M., Sessler, J. L., and Arambula, J. F. (2021). Covalent and Non-covalent Albumin Binding of Au(i) Bis-NHCs via postsynthetic Amide Modification. Chem. Sci. 12, 7547-7553. doi:10.1039/d1sc01055g

Sheng, Z., Hu, D., Zheng, M., Zhao, P., Liu, H., Gao, D., et al. (2014). Smart Human Serum Albumin-Indocyanine Green Nanoparticles Generated by Programmed Assembly for Dual-Modal Imaging-Guided Cancer Synergistic Phototherapy. ACS Nano 8, 12310-12322. doi:10.1021/nn5062386

Song, F., Liu, B., and Tang, B. Z. (2020). Celebration of the 20th Anniversary of Aggregation-Induced Emission with Research Highlights from Royal Society of Chemistry Journals. J. Mater. Chem. C 8, 7972-7974. doi:10.1039/d0tc90119a

Steinbrueck, A., Sedgwick, A. C., Brewster, J. T., Yan, K.-C., Shang, Y., Knoll, D. M., et al. (2020). Transition Metal Chelators, Pro-chelators, and Ionophores as Small Molecule Cancer Chemotherapeutic Agents. Chem. Soc. Rev. 49, 3726-3747. doi:10.1039/c9cs00373h

Steinbrueck, A., Sedgwick, A. C., Han, H.-H., Zhao, M. Y., Sen, S., Huang, D.-Y., et al. (2021). In Vitro studies of Deferasirox Derivatives as Potential OrganelleTargeting Traceable Anti-cancer Therapeutics. Chem. Commun. 57, 5678-5681. doi:10.1039/d0cc08156f
Thomas, B., Yan, K.-C., Hu, X.-L., Donnier-Maréchal, M., Chen, G.-R., He, X.-P., et al. (2020). Fluorescent Glycoconjugates and Their Applications. Chem. Soc. Rev. 49, 593-641. doi:10.1039/c8cs00118a

Tian, H., Sedgwick, A. C., Han, H.-H., Sen, S., Chen, G.-R., Zang, Y., et al. (2021a). Fluorescent Probes for the Imaging of Lipid Droplets in Live Cells. Coord. Chem. Rev. 427, 213577. doi:10.1016/j.ccr.2020.213577

Tian, X., Murfin, L. C., Wu, L., Lewis, S. E., and James, T. D. (2021b). Fluorescent Small Organic Probes for Biosensing. Chem. Sci. 12, 3406-3426. doi:10.1039/ d0sc06928k

Wei, X., and Xunbin, G. (2021). Optical Imaging in Human Disease and Biological Research. Singapore: Springer.

Wu, D., Sedgwick, A. C., Gunnlaugsson, T., Akkaya, E. U., Yoon, J., and James, T. D. (2017). Fluorescent Chemosensors: the Past, Present and Future. Chem. Soc. Rev. 46, 7105-7123. doi:10.1039/c7cs00240h

Wu, L., Huang, C., Emery, B. P., Sedgwick, A. C., Bull, S. D., He, X.-P., et al. (2020). Förster Resonance Energy Transfer (FRET)-based Small-Molecule Sensors and Imaging Agents. Chem. Soc. Rev. 49, 5110-5139. doi:10.1039/c9cs00318e

Wu, L., Huang, J., Pu, K., and James, T. D. (2021a). Dual-locked Spectroscopic Probes for Sensing and Therapy. Nat. Rev. Chem. 5, 406-421. doi:10.1038/s41570-02100277-2

Wu, L., Liu, J., Li, P., Tang, B., and James, T. D. (2021b). Two-photon SmallMolecule Fluorescence-Based Agents for Sensing, Imaging, and Therapy within Biological Systems. Chem. Soc. Rev. 50, 702-734. doi:10.1039/d0cs00861c

Wu, L., Sedgwick, A. C., Sun, X., Bull, S. D., He, X.-P., and James, T. D. (2019). Reaction-Based Fluorescent Probes for the Detection and Imaging of Reactive Oxygen, Nitrogen, and Sulfur Species. Acc. Chem. Res. 52, 2582-2597. doi:10.1021/acs.accounts.9b00302

Yan, K.-C., Sedgwick, A. C., Zang, Y., Chen, G.-R., He, X.-P., Li, J., et al. (2019). Sensors, Imaging Agents, and Theranostics to Help Understand and Treat Reactive Oxygen Species Related Diseases. Small Methods 3, 1900013. doi:10.1002/smtd.201900013

Yang, M., Huang, J., Fan, J., Du, J., Pu, K., and Peng, X. (2020). Chemiluminescence for Bioimaging and Therapeutics: Recent Advances and Challenges. Chem. Soc. Rev. 49, 6800-6815. doi:10.1039/d0cs00348d

Yeo, C. I., Ooi, K. K., and Tiekink, E. R. T. (2018). Gold-Based Medicine: A Paradigm Shift in Anti-Cancer Therapy? Molecules 23, 1410. doi:10.3390/molecules23061410

Zhang, L., Duan, D., Liu, Y., Ge, C., Cui, X., Sun, J., et al. (2014). Highly Selective Off-On Fluorescent Probe for Imaging Thioredoxin Reductase in Living Cells. J. Am. Chem. Soc. 136, 226-233. doi:10.1021/ja408792k

Zhang, Z., Song, W., Su, J., and Tian, H. (2020). Vibration-Induced Emission (VIE) of N,N '-Disubstituted-Dihydribenzo $[a, c]$ phenazines: Fundamental Understanding and Emerging Applications. Adv. Funct. Mater. 30, 1902803. doi:10.1002/adfm.201902803

Zhou, X., Li, H., Shi, C., Xu, F., Zhang, Z., Yao, Q., et al. (2020). An APN-Activated NIR Photosensitizer for Cancer Photodynamic Therapy and Fluorescence Imaging. Biomaterials 253, 120089. doi:10.1016/j.biomaterials.2020.120089

Zwicker, V. E., Oliveira, B. L., Yeo, J. H., Fraser, S. T., Bernardes, G. J. L., New, E. J., et al. (2019). A Fluorogenic Probe for Cell Surface Phosphatidylserine Using an Intramolecular Indicator Displacement Sensing Mechanism. Angew. Chem. Int. Ed. 58, 3087-3091. doi:10.1002/anie.201812489

Conflict of Interest: The authors declare that the research was conducted in the absence of any commercial or financial relationships that could be construed as a potential conflict of interest.

Publisher's Note: All claims expressed in this article are solely those of the authors and do not necessarily represent those of their affiliated organizations, or those of the publisher, the editors and the reviewers. Any product that may be evaluated in this article, or claim that may be made by its manufacturer, is not guaranteed or endorsed by the publisher.

Copyright (C) 2021 Yan, Steinbrueck, Sedgwick and James. This is an open-access article distributed under the terms of the Creative Commons Attribution License (CC $B Y$ ). The use, distribution or reproduction in other forums is permitted, provided the original author(s) and the copyright owner(s) are credited and that the original publication in this journal is cited, in accordance with accepted academic practice. No use, distribution or reproduction is permitted which does not comply with these terms. 